\title{
An Updated Distance to the Cygnus Loop based on Gaia Early DR3
}

\author{
Robert A. Fesen, ${ }^{1 \star}$ Kathryn E. Weil, ${ }^{2}$ Ignacio Cisneros, ${ }^{1}$ William P. Blair, ${ }^{3}$ and John C. Raymond, ${ }^{4}$ \\ ${ }^{1}$ Department of Physics and Astronomy, 6127 Wilder Laboratory, Dartmouth College, Hanover, NH 03755, USA \\ ${ }^{2}$ Department of Physics and Astronomy, Purdue University, 525 Northwestern Avenue, West Lafayette, IN 47907 USA \\ ${ }^{3}$ The Henry A. Rowland Department of Physics \& Astronomy, Johns Hopkins University, 3400 N. Charles Street, Baltimore, MD, 21218, USA \\ ${ }^{4}$ Harvard-Smithsonian Center for Astrophysics, 60 Garden St., Cambridge, MA 02138, USA
}

Accepted 2021 July 13. Received 2021 July 11; in original form 2021 June 26

\begin{abstract}
We present a revised distance to the Cygnus Loop supernova remnant of $725 \pm 15$ pc based on Gaia Early Data Release 3 parallax measurements (EDR3) for several stars previously found to be located either inside or behind the supernova based on the presence of high-velocity absorption lines in their spectra. This revised distance estimate and error means the Cygnus Loop remnant now has an estimated distance uncertainty comparable to that of its $\simeq 18 \mathrm{pc}$ radius.
\end{abstract}

Key words: ISM:supernova remnants - stars: distances

\section{INTRODUCTION}

The Galactic supernova remnant (SNR) G74.0-8.5, commonly known as the Cygnus Loop or Veil Nebula, is thought to be a middleage remnant with an estimated age $\approx 2 \times 10^{4}$ yr. The distance to the Cygnus Loop is a key parameter in estimating its mass, energy and evolutionary state, as well as its shock velocities determined from filament proper motions. The most widely adopted value for the Cygnus Loop's distance had long been 770 pc based on a kinematic investigation by Minkowski (1958). However, subsequent distance estimates have ranged between 500 to 1000 pc (Blair et al. 2009; Salvesen et al. 2009; Medina et al. 2014; Raymond et al. 2015). See Fesen et al. (2018a) for a full review.

The most recent distance estimate is based on $\mathrm{Na}$ I 5890, 5896 $\AA$ and Ca II $3934 \AA$ absorption lines with velocities ranging from -150 to $+240 \mathrm{~km} \mathrm{~s}^{-1}$ in the spectra of stars with projected locations toward the remnant (Fesen et al. 2018b). The presence of both red- and blue-shifted absorptions means the stars must be located behind the Cygnus Loop's expanding front and rear hemispheres. Combining Gaia Data Release 2 (DR2) parallax measurements (Gaia Collaboration et al. 2018) for three such stars plus the estimated distance for a B7 star BD+31 4224 located along the remnant's northwestern limb (Fesen et al. 2018a), led to an estimated a distance to the Cygnus Loop's center of $735 \pm 25 \mathrm{pc}$ and a model where the remnant's eastern side was tilted toward us. However, this result involved simple parallax inversion and not a Bayesian analysis (Luri et al. 2018).

Here we present an updated Cygnus Loop distance using Gaia Early Data Release 3 (EDR3; Gaia Collaboration et al. 2021) of these same stars with parallaxes less than half the uncertainty of DR2 parallaxes for stars with $\mathrm{G}$ magnitudes between 9 and 12 (Lindegren et al. 2021). We adopt the star naming convention of Fesen et al. (2018b)

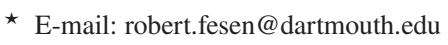

along with their conclusions about the locations of these stars with respect to the Cygnus Loop remnant.

\section{GAIA EARLY DR3 PARALLAX MEASUREMENTS}

Table 1 lists Gaia EDR3 parallaxes and parallax errors for three stars, dubbed X, Y, and Z, with projected locations along the eastern half of the Cygnus Loop that Fesen et al. (2018b) found to exhibit high-velocity $\mathrm{Na} 1$ and $\mathrm{Ca} 2$ absorption components. The table also includes Gaia parallaxes for the sdO star KPD 2055+3111 found by Blair et al. (2009) which also exhibited high-velocity absorption features attributed to the Cygnus Loop, and the B7 V star BD+31 4224 proposed by Fesen et al. (2018a) to physically lie inside or physical contact with the remnant's shocks along the northwestern limb. Coordinates and V magnitudes for these five stars are listed along with the nominal geometric parallax distances $(\mathrm{d}=$ $1 / p$ ) and corrected distance estimates following a probabilistic analysis as described by Bailer-Jones (2015) and tabulated for EDR3 by Bailer-Jones et al. (2021) using a three-dimensional model of the Galaxy and a direction-dependent prior of distance.

\section{DISCUSSION}

Since the star KPD $2055+3111$ and Star Z lie at corrected EDR3 of distances of 819 and 835 pc, respectively, they must lie well behind the the Cygnus Loop remnant based on the appearance of SNR related high-velocity absorption lines, similar to those seen in the much closer stars, $\mathrm{X}$ and $\mathrm{Y}$. Hence, they are not particularly helpful in constraining the remnant's distance except in setting a firm maximum distance of $\simeq 800 \mathrm{pc}$. We note that a previous search for high-velocity absorption components associated with the remnant failed for stars with distances less than $\sim 600 \mathrm{pc}$ (Welsh et al. 2002) which sets a rough lower limit.

Adopting the situation described by Fesen et al. (2018a) where the 
Table 1. Stars Exhibiting High-Velocity Absorption Features Associated with the Cygnus Loop Supernova Remnant

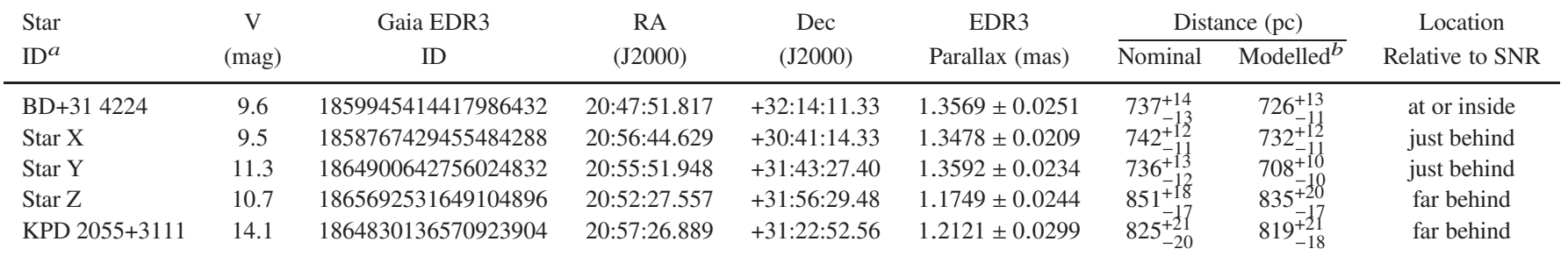

${ }^{a}$ References: BD+31 4224: Fesen et al. (2018a); X,Y,Z: Fesen et al. (2018b); KPD 2055 +3111: Blair et al. (2009)

${ }^{b}$ Direction-dependent distances from Bailer-Jones et al. (2021).

stellar winds from the B7 star BD+31 4224 have physically interacted with the remnant's expanding shock front implies this star lies either inside or is in immediate contact with the remnant's expanding shock front. This firmly anchors the remnant to a distance close to that estimated for this star of $726_{-11}^{+13}$. However, due to the star's half chord length of $\sim 5.4 \mathrm{pc}$ to the remnant center in the star's sight line along the remnant's northwestern limb, this increases the Cygnus Loop's center distance uncertainty range by roughly $5 \mathrm{pc}$ to $726_{-16}^{+18}$ pc, corresponding to 710 thru $744 \mathrm{pc}$.

Since blue and red absorption Na 1 lines from the Cygnus Loop's front and rear expanding shells were observed in the spectra of both Stars X and Y, their EDR3 estimated distances can be used to set additional limits on the remnant's distance. Ignoring the Cygnus Loop's southern blowout region and adopting a center near RA $(\mathrm{J} 2000)=$ 20:51:11.2, Dec $(J 2000)=+31: 03: 10$ for the remnant's main emission structure, its $2.88^{\circ}$ angular diameter implies a physical radius of $\simeq 18.5 \mathrm{pc}$ at the Fesen et al. (2018b) estimated distance of $735 \mathrm{pc}$. Stars X and Y lie approximately $87 \%$ and $84 \%$, respectively, of the remnant's radius away from its center, meaning the half chord lengths along their sight lines from the remnant's perpendicular center line to its rear hemispheric edge are $9.3 \mathrm{pc}$ and $10.2 \mathrm{pc}$, respectively. This effectively decreases the possible location of the Cygnus Loop's center distance using the distances to these background stars, namely $723 \pm 12$ pc for Star X and $698 \pm 11$ pc for Star Y.

The smaller distance to Star $\mathrm{Y}$ is especially restrictive since we know it has to lie completely behind the remnant. Even assuming a tilt of the remnant's eastern region toward us as described by Fesen et al. (2018b), remnant center distances above $\simeq 740 \mathrm{pc}$ seem unlikely given Star Y's maximum estimated distance of $718 \mathrm{pc}$. We are thus left with a small range of possible distances to the B7 star and hence the Cygnus Loop's center of 710 to $740 \mathrm{pc}$ or $725 \pm 15 \mathrm{pc}$.

This new distance estimate can be combined with proper motions to determine the speeds of radiative shock waves in the western Cygnus Loop, and those speeds yield good agreement between models and observed spectra (Raymond et al. 2020). However, the tight constraint on distance exacerbates a problem. Proper motions of the northern nonradiative shocks and a distance of $725 \mathrm{pc}$ imply shock speeds that are a bit too small to match the thermal energies given by the electron temperatures from X-rays and the proton temperatures from the $\mathrm{H} \alpha$ profiles (Salvesen et al. 2009; Medina et al. 2014). New proper motion measurements of the northern filaments (Milanovic et al. 2019) generally agree with the proper motions of Salvesen et al. (2009) and the shock speeds derived by Medina et al. (2014). Consequently, the problem might result either from errors in proper motion measurements or from an inaccuracy in the models that relate the $\mathrm{H} \alpha$ line width to the proton temperature.

This slightly shorter distance estimate than the $735 \pm 25 \mathrm{pc}$ of Fesen et al. (2018b) does not appreciably alter any of the Cygnus Loop's properties derived in that paper. But it does mean that the
Cygnus Loop remnant now has an estimated distance uncertainty comparable to that of its $\simeq 18 \mathrm{pc}$ radius, a remarkable result and a testament to the power of Gaia's accurate parallax measurements. But given that this estimate depends heavily on the assumption that the stellar winds from the B7 star BD+31 4224 have physically interacted with the Cygnus Loop's expanding shocks thereby firmly anchoring the remnant's distance, finding additional stars at varying distances toward the Cygnus Loop showing high-velocity absorption lines in their spectrum would be valuable in providing independent tests of this result.

\section{ACKNOWLEDGEMENTS}

This work made use of data from the European Space Agency mission Gaia (https://www. cosmos.esa.int/gaia), processed by the Gaia Data Processing and Analysis Consortium (DPAC, https://www. cosmos.esa.int/web/gaia/dpac/consortium). Funding for the DPAC has been provided by national institutions, in particular the institutions participating in the Gaia Multilateral Agreement.

\section{DATA AVAILABILITY}

Parallax data are available through the Gaia archive website.

\section{REFERENCES}

Bailer-Jones C. A. L., 2015, PASP, 127, 994

Bailer-Jones C. A. L., Rybizki J., Fouesneau M., Demleitner M., Andrae R., 2021, AJ, 161, 147

Blair W. P., Sankrit R., Torres S. I., Chayer P., Danforth C. W., 2009, ApJ, 692,335

Fesen R. A., Neustadt J. M. M., Black C. S., Milisavljevic D., 2018a, MNRAS, 475, 3996

Fesen R. A., Weil K. E., Cisneros I. A., Blair W. P., Raymond J. C., 2018b, MNRAS, 481, 1786

Gaia Collaboration Brown A. G. A., Vallenari A., et al. 2018, A\&A, 616, A1

Gaia Collaboration Brown A. G. A., Vallenari A., et al. 2021, A\&A, 650, C3

Lindegren L., et al., 2021, A\&A, 649, A2

Luri X., et al., 2018, A\&A, 616, A9

Medina A. A., Raymond J. C., Edgar R. J., Caldwell N., Fesen R. A., Milisavljevic D., 2014, ApJ, 791, 30

Milanovic N., Vucetic M., Onic D., Urovsevic D., Raymond J., 2019, in Supernova Remnants: An Odyssey in Space after Stellar Death II. p. 127

Minkowski R., 1958, Reviews of Modern Physics, 30, 1048

Raymond J. C., Edgar R. J., Ghavamian P., Blair W. P., 2015, ApJ, 805, 152

Raymond J. C., Slavin J. D., Blair W. P., Chilingarian I. V., Burkhart B., Sankrit R., 2020, ApJ, 903, 2 
Salvesen G., Raymond J. C., Edgar R. J., 2009, ApJ, 702, 327

Welsh B. Y., Sallmen S., Sfeir D., Lallement R., 2002, A\&A, 391, 705

This paper has been typeset from a $\mathrm{T}_{\mathrm{E}} \mathrm{X} / \mathrm{LT} \mathrm{E} \mathrm{X}$ file prepared by the author. 